\title{
Newer Lights in Management of Snake Bite Cellulitis, Surgical Vs. Medical.
}

\author{
Dr.S.Ganeshbabu,M.S ${ }^{1}$, Dr.T.Ravikumar,M.D ${ }^{2}$, Dr.Kesavalingam, $\mathrm{MS}^{3},{ }^{3}$, \\ Dr.N.Tamilselvan,M.S ${ }^{4}$, Dr.P.Saravanan,M.D ${ }^{5}$, Dr. Suresh,M.D ${ }^{6}$ \\ Dr.Palaniappan'M.D ${ }^{6}$, Dr.S.T.Jaya sudha ${ }^{7}$ \\ ${ }^{1}$ Professor of Surgery, Govt Theni Medical College,Theni, \\ ${ }^{2}$ Professor and Head, Department of Medicine, Govt.Medical College \& ESI Hospital, Coimbatore \\ ${ }^{3}$ Professor of Surgery Gmkmch Salem ${ }^{4}$ Prof of Surgery GMC \& ESICH, CBE. ${ }^{5}$ Asst Professor Of Medicine \\ Mmc Madurai 6 Asst Professor Of Medicine, GMC \& ESIC, Cbe ${ }^{7}$ Postgraduate in surgery Gmkmch Salem
}

\begin{abstract}
Incidences of Snake bite are inseparable from human life, especially in rural areas because the reptiles live both in domestic and in wild. Snake bite is of great public health interest not only because it volves huge financial Commitment from the government but also due to its morbidity and mortalityAs for the medical fraternity is concern the responsibility lies in reducing the morbidity, mortality as well as reducing the xpenses. This can be achieved by using appropriate dose of ASV in appropriate time and early fasciotomy for the needy patients at the right time There are many guidelines available for medical management were as no proper guidelines available for managing the snakebite induced cellulitis and compartmental syndrome we have attempted to propose a few guide lines for the management of the same

Material And Methods: This is a prospective observational study in a tertiary medical care hospital in the western part of Tamil Nadu between Jan 2013 to Dec 2015 with 1224 patients all are presented with history of snake bite and signs of envenomation

Inclusion Criteria: All the patients with features of envenomation,Patients with features of impending gangrene. Exclusion: patients with co-morbidity like diabetes and hypertension Patients on aspirin, and bleeding diathesis

Study Period: all patients admitted between Jan 2013 to Dec 2015

Aim Of The Study: The aim of the study is to study and compare the outcome of intensive medical management with ASV and medicines including analgesic verses surgical are useful in reducing the morbidity due to snake bite induced cellulites. The presently available polyvalent ASV, one effective against bites due to common neurotoxic and hemotoxic snakes. Theoretically, it would appear that patients with more severe envenomation need higher doses of ASV for effective neutralization of circulating snake venom but practically it is not many of the times we need surgical intervention but there are no universal acceptable guidelines available hence on this study will help to form a guide lines

Results: Intensive treatment with ASV and appropriate management of cellulitis with appropriate antibiotics will reduce the morbidity, but early bullae formation, discoloration, compartmental syndrome, gangrene even in presence of abnormal coagulation profile can be effectively managed with surgical decompression with transfusion of FFP.with medical management
\end{abstract}

Conclusions: Fasciotomy should be done at the earliest in fingers and toes, after aggressive ASV Fasciotomy for proximal parts of the limbs should be done once we see a bleb.

When the fasciotomy has to cross a joint follow $z$ pattern while incising. Trunk fasciotomy can be postponed until gangrenous changes are evident

Keywords: ASV,Snake bite cellulitis, compartment syndrome, fasciotomy

\section{Introduction}

Primarily snakebites carry the consequences of envenomation leading to lesion formation at the bite site along with extensive tissue necrosis. This dead tissue can acquire secondary infection from bacteria coming from the snake's mouth inoculated at the time of the bite. Until recently, very little was known about the spectrum of bacteria responsible for wound infections in snakebite patients.

The proteolytic properties of snake venom cause extensive tissue destruction and devitalisation, thus predisposing the wound to bacterial infection from the snake's indigenous oral flora. Although 
bacteria are a major cause of wound infection in snakebite patients, the role of prophylactic antibiotics to prevent their formation is debatable.

1. Ulcer following snakebite

\section{Surgical Issues Observed In Snake Bite Cases Are}

2. Necrosis of the skin and underlying tissues

3. Gangrene of the toes and fingers

4. Debridement of necrotic tissues

5. The Compartment syndrome and others

In our study majority of the patients presented with cellulities responded to early aggressive medical management with ASV,(85\%) if cellulitis presents within two hours 5vials of ASV when it cross one joint 5 vials of ASV added (eg. up to wrist 5 vials upto elbow 10 vials upto shoulder 15 vials) with anti oedema measures and analgesics preferably paracetamal The appearance of an immobile, tensely-swollen, cold and apparently pulseless snake-bitten limb may suggest to surgeons the possibility of increased intra-compartmental pressure, especially if the digital pulp spaces or the anterior tibial compartment are involved.

Swelling of envenomed muscle within such tight fascial compartments could result in an increaseintissue pressure above the venous pressure, resulting in ischaemia.

However, the classical signs of an intra-compartmental pressure syndrome may be difficult to assess snake-bite victims and many unnecessary, dangerous and debilitating fasciotomies are performed, especially where surgeons rather than physicians have the primary responsibility for managing snake-bite cases.

\section{Clinical features of a compartmental syndrome}

- Disproportionately severe pain.

- Weakness of intra compartmental muscles.

- Pain on passive stretching of intra compartmental muscles.

- Hypo aesthesia of areas of skin supplied by nerves running through the compartment.

- Obvious tenseness of the compartment on palpation.

Detection of arterial pulses by palpation or Doppler ultrasound probes, does not exclude intracompartmental ischemia. The most reliable test is to measure intra compartmental pressure directly through a cannula introduced into the compartment and connected to a pressure transducer or manometer.

In orthopaedic practice, intracompartmental pressures exceeding $40 \mathrm{mmHg}$ (less in children) may carry a risk of ischaemic necrosis (e.g.Volkmann'sischaemia or anterior tibial compartment syndrome). However,envenomed muscle may not be saved by fasciotomy (Animal studies have suggested that muscle sufficiently envenomed and swollen to cause intra-compartmental syndromes, may already be irreversibly damaged by the direct effects of the venom).

In any case, fasciotomy should not be contemplated until haemostatic abnormalities have been corrected, otherwise the patient may bleed to death not only in reversing coagulopathy, antivenom may also be helpful in reducing severe limb oedema. However, corticosteroids are not effective in ameliorating local effects of envenoming and, since they carry the risk of side-effects, they should not be used.

Early treatment with antivenom remains the best way of preventing irreversible muscle damage. Criteria for fasciotomy in snake-bitten limbs Haemostatic abnormalities have been corrected (antivenom with or without clotting factors) Clinical evidence of an intracompartmental syndrome Intracompartmental pressure $>40$ $\mathrm{mmHg}$ (in adults)

\section{Rehabilitation}

In patients with severe local envenoming, the limb should be maintained in a functional position. For example, in the leg, equinus deformity of the ankle should be prevented by application of a back slab (or) Ankle brace. Functional effects of local envenoming range from persistent stiffness and indurations due to sclerosis of veins, lymphatics and tissue planes through which the venom has spread, to severe deformity, tissue loss especially dermo-necrosis, and requiring skin grafting and gangrene requiring debridement and amputation.

Restoration of normal function in the bitten part should be started by simple exercises while the patient is still in hospital. After the patient has been discharged from hospital rehabilitation is rarely supervised but relatives can be instructed and given a time table of rehabilitation activities Conventional physiotherapy may accelerate functional recovery of the bitten limb. 


\section{Reviews Of Literature}

A retrospective review of all cases of wound infections secondary to snakebite was conducted at Jipmer Hospital, Pondicherry, India, for the period January 2003 to October 2008. A total of 43 infected snakebite cases (31 male and 12 females: $86 \%$ adults) were included in this study. Monomicrobial infection was present in 33 cases, whereas mixed infection by two bacteria was observed in 10 cases. The oral flora of snake comprises a wide range of aerobic and anaerobic micro-organisms, especially the fecal Gram-negative rods, because their prey usually defecate while being ingested. Culture of fangs, fang sheaths, and venom of various snakes such as bothrops, vipers, rattlesnakes and najanaja, have shown heavy colonization with many bacteria, including members of Enterobacteriaceae including Morganella spp., Escherichia coli, Group D streptococci, Aeromonas spp., and anaerobes such as Clostridium spp.

Table 1. Bacteria isolated from infected wounds following snakebite

\begin{tabular}{|l|c|}
\hline Bacteria isolated $(\mathbf{n}=\mathbf{5 3})$ & Number \\
\hline Gram-positive bacteria $(\mathbf{n = 2 8})$ & \\
\hline Staphylococcus aureus & 17 \\
\hline Coagulase negative staphylococcus & 5 \\
\hline Enterococcus faecalis & 4 \\
\hline Streptococcus spp & 2 \\
\hline Gram-negative bacteria $(\mathbf{n}=\mathbf{2 5})$ & \\
\hline Escherichia coli & 8 \\
\hline Klebsiella pneumoniae & 4 \\
\hline Proteus spp. & 3 \\
\hline Morganella morganii & 3 \\
\hline Pseudomonas aeruginosa & 3 \\
\hline Acinetobacter spp & 2 \\
\hline Enterobacter spp & 2 \\
\hline
\end{tabular}

A retrospective study from Zimbabwe involving cases of cellulitis secondary to snakebite demonstrated that prophylactically the most frequently used antibiotics were drugs in the penicillin family.

Another study from Southern Africa suggested that if antibiotics are to be used empirically in patients with snakebite, then members of Staphylococcus spp. and Gram-negativebacteria such as Enterobacteriaceae must be covered Studies from Saudi Arabia and Eastern Ecuador have reported that ampicillin alone or in combination with another antibiotic were most commonly used for management of snakebites

Another retrospective descriptive study done from January to August 2011 to identify the pattern of antibiotics used following snake envenomation in a tertiary care hospital of Kerala

A total of 313 case records with diagnosis of snake bite were studied of which $58.1 \%(n=182)$ were males and $41.9 \%(n=131)$ females. Majority of patients fell in the 1-45 years age group as shown in [Table/Fig-1]. The mean age was $37.58 \pm 14.54$ years.

About $94.6 \%(\mathrm{n}=296)$ received antibiotics, of which $24.32 \%$ had evidence of local and $10.81 \%$ had systemic envenomation, while $64.86 \%(\mathrm{n}=192)$ were patients with no envenomation.

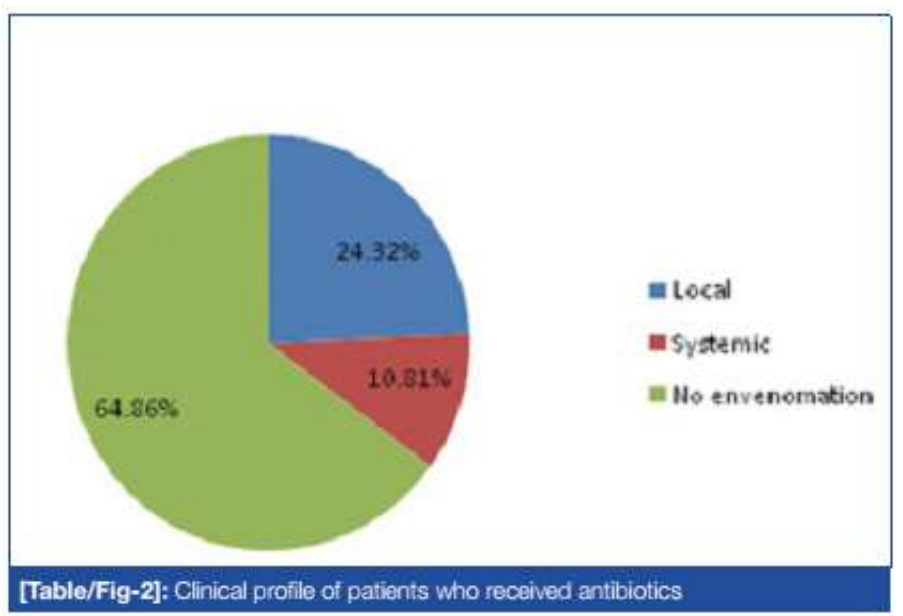


The study concluded that there was no statistically significant (chi-square $=5.237, \mathrm{p}=0.07$ ) pattern in using single or combination antibiotics with types of envenomation, in patients with no signs of envenomation who received antibiotics.

Some authors have suggested that before commencing antibiotic therapy culture and sensitivity tests have to be performed [13]. Garg et al., opined that the performance of bacterial culture and antimicrobial sensitivity in each and every patient was a challenge and implementing it was impractical especially in the rural and tribal settings The study concluded and justified the prophylactic use of antibiotics following snake bite for the concerns of secondary infections Since done in the setting of a developing country

\section{HBO Therapy:}

A study done to evaluate Hyperbaric oxygen therapy(HBO) in outcome of snake envenomation by Pradeoth Korambayi lMukundan et al in tertiary centre experience in Kerala.Of a total 395 patients, 23 presented with cellulitis, 7 with compartment syndrome, and 17 for the management of soft tissue cover over the extremities. Of the 47 patients, 30 involved the lower extremity and rest involved the upper extremity. All patients were subjected to HBO therapy as an adjunct. Six patients required flap cover: There was no need for fasciotomy among patients who suffered impending compartment syndrome.

The study discussed ASV administration can reduce progression of the initial tissue damage, but it cannot reverse local tissue damage such as tissue edema, inflammation, compartment syndrome, and necrosis.

The inflammation induced by snake envenomation often mimics infection, true bacterial cellulitis is uncommon, and only affects $3 \%$ of snakebites. Tissues at the point of envenomation may be nonviable regardless of intervention.

The adjacent zone consists of variably injured tissues that may recover if the process of inflammation is reduced. Most therapeutic manoeuvres are focused on this penumbra of tissue, in an attempt to maximize recovery of the injured marginal tissue. Finally, an outer zone of minimally injured tissues that are not subject to primary injury may be at risk from the processes of secondary injuryresulting from the delayed, physiological inflammatory responses to snake bite injury.

The study suggested HBO therapy can reduce the penumbra of cells at risk for delayed necrosis and secondary ischemia in snakebite injury patients with early compartment syndrome, breathing of $100 \%$ oxygen under increased ambient pressure prevents reperfusion injury, reduces tissue edema, and reverses sub lethal tissue damage. Vasoconstriction reduces edema and tissue swelling while ensuring adequate oxygen delivery in snakebite wounds.

Six sessions are minimum given to attain a tangible decrease in swelling. Assessment of sensory loss may not be possible in small children, and hence we determine the treatment endpoint based on the decrease in girth of the limb and also the movement of the toes. HBO therapy after surgical debridement, skin grafting or flap reduces edema of the inflamed operation site.

When compartment syndrome occurs in snakebite injuries, there is controversy as to whether or not fasciotomy is required .In a case series conducted by Tanen et al., only 8/236 (3.4\%) of patients received a fasciotomy or digital dermotomy for compartment syndrome. Measurement of compartment pressures prior to fasciotomy is always recommended. Recent literature indicates that an increase of intra-compartmental pressures of up to $30-45 \mathrm{mmHg}$ is an absolute indication for fasciotomy.

Unfortunately, measurement of intra-compartmental pressure is not always possible in a number of medical centres in India, and most of the diagnoses of compartment syndrome are made on clinical grounds alone. Anz et al. reported that $21.2 \%$ of all poisonous snake bites involve fingers. Fingers have small compartments with its small diameters, and the elastic limit of the skin can be rapidly reached. Compartment pressure measurement may not be feasible in cases of digital envenomation, and the diagnosis of compartment syndrome can only be made on clinical grounds. Some authors support early fasciotomy in the treatment of all cases of snake bite envenomation. Fasciotomies are not without complication, and may result in disfiguring scars, contractures, nerve damage, leading to significantly lengthening of treatment.

\section{Another study done by Darracq et al and colleaguesfavoured against routine fasciotomies.}

Out of 105, Fasciotomy was performed in 28 cases $(27 \%)$. There was no statistically significant difference $(\mathrm{p}>0.05)$ between groups in age, gender, bite site, SSS, and total number of vials of antivenom administered. Only 2 of $28(7 \%)$ had compartment pressure measurements. Patients undergoing fasciotomy spent an additional 2 days in the hospital.

In another study Cumpston et al concluded fasciotomy either has no beneficial effect or worsens myonecrosis.Mazer-Amirshahi et al and colleagues concluded early and aggressive treatment with antivenin may have avoided invasive fasciotomy, and its use should be considered in patients with copperhead envenomation and significantly elevated compartment pressures. 
GargAet al and colleagues published that Staphylococcus aureus (32\%) was the most common isolate followed by Escherichia coli (15\%); monomicrobial infections were more frequent than polymicrobial infections. The majority of the isolates were antibiotic sensitive. Ciprofloxacin, an oral drug covering both Gram-positive and Gram-negative isolates, was the most frequently prescribed antibiotic. They suggested their study would helpin deciding the empirical antibiotic therapy in cases of wound infection secondary to snakebite in regions of Southeast Asia.

Jung Ho Rha et al and colleagueson their research publication reiterated that immediate debridement is no longer the treatment of choice in managing snake bites; rather, the current standard of care is administration of antivenom followed by delayed debridement.They also quoted a study using an electron microscope observed viable tissues buried inside necrotized muscles of the snakebite wound, providing additional evidence for preemptiveantivenom use followed by delayed debridement.

In WHO guidelines for the management of snake-bites, interference with the wound (incisions made with an unsterilized razor blade/knife etc) creates a risk of secondary bacterial infection and justifies the use of immediate broad spectrum antibiotics (e.g. amoxycillin or a cephalosporin plus a single dose of gentamicin plus metronidazole) and tetanus prophylaxis. Infection at the time of the bite with organisms from the snake's venom and buccal cavity is a problem with some species such as the Malayan pit viper (Theakston et al., 1990) but prophylactic antibiotics were not effective in a controlled study in Brazil (Jorge et al., 2004)

In any case, fasciotomy should not be contemplated until haemostatic abnormalities have beencorrected, otherwise the patient may bleed to death. Not only in reversing coagulopathy, antivenom may also be helpful in reducing severe limb oedema (Rojnuckarin et al., 2006). However, corticosteroids are not effective in ameliorating local effects of envenoming and, since they carry the risk of side-effects, they should not be used (Reid et al., 1963; Nuchprayoon et al., 2008).

\section{They also published results of unnecessary fasciotomies in snake bite victim inThailand.}

(a) Profuse bleeding in patients with mild local envenoming but severe coagulopathy following a bite by green pit viper

(b) Residual skin loss and exposure of tendons following fasciotomy for mild local envenoming in a patient bitten by a green pit viper

(c) Persistent bleeding for 10 days, resulting in haemorrhagic shock despite transfusion of 20 units of blood, in a victim of Malayan pit viper bite in whom fasciotomy was performed before adequate antivenom treatment had been given to correct the coagulopathy

\section{Conclusion}

Early and aggressive treatment with antivenin will prevent invasive fasciotomy.No definitive guidelines are available for prophylactic treatment for snake bite with envenomation. The spectrum of bacteria from the venom and oral cavities of snakes vary with geographic area as well as with the species and the oral health of the snake, and these factors cannot easily be extrapolated to snakes in rest of the world. It is difficult to perform bacterial culture and antimicrobial susceptibility for every patient of snakebite, particularly for those living in rural and tribal areas; hence regional studies are required to identify the spectrum of bacteria and their antibiotic susceptibility pattern.

However, it is strongly suggested that clinical specimens for culture should be collected before commencing antibiotic therapy for cases of snakebite associated with cellulitis, abscess, gangrene or bulla.

\section{Figures:}

Fig 1:

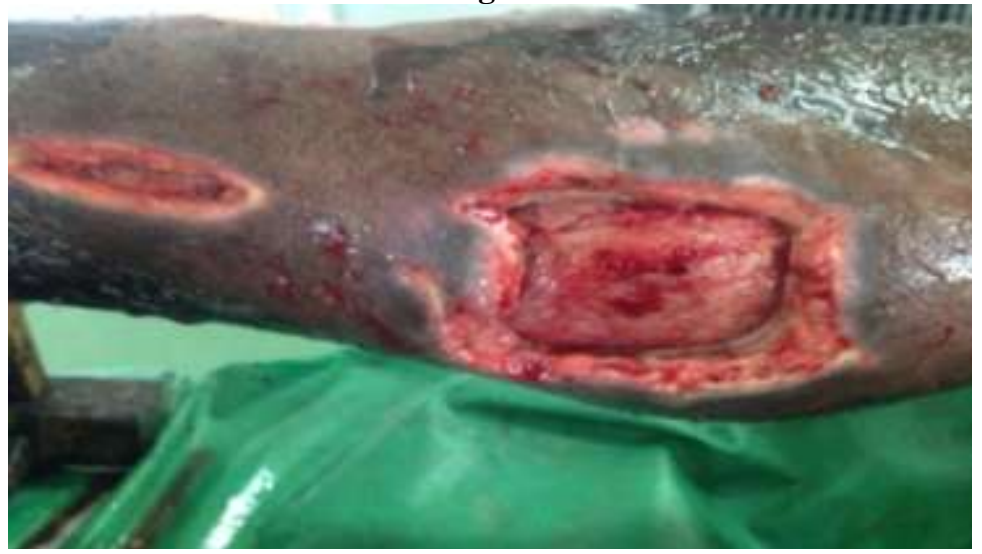




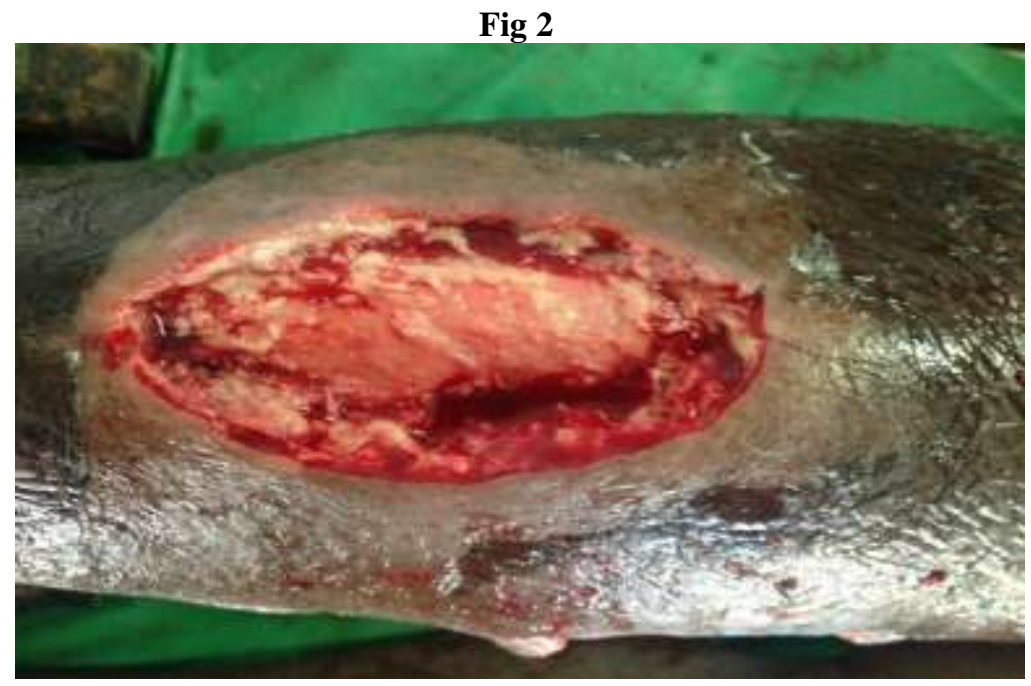

Fig 3

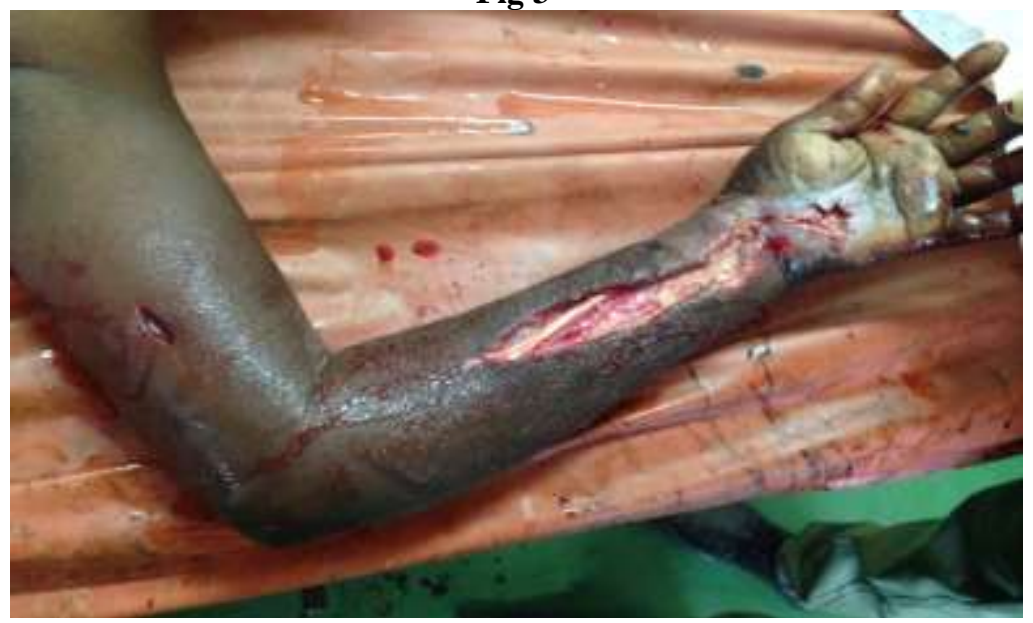

Fig 4:

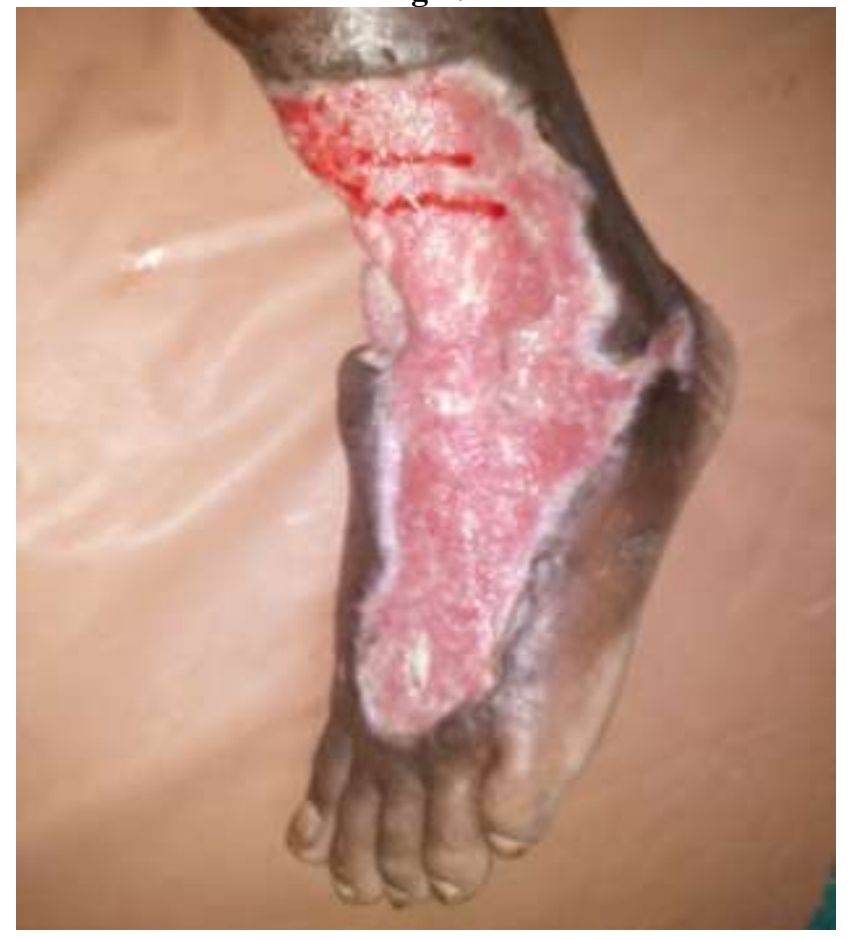



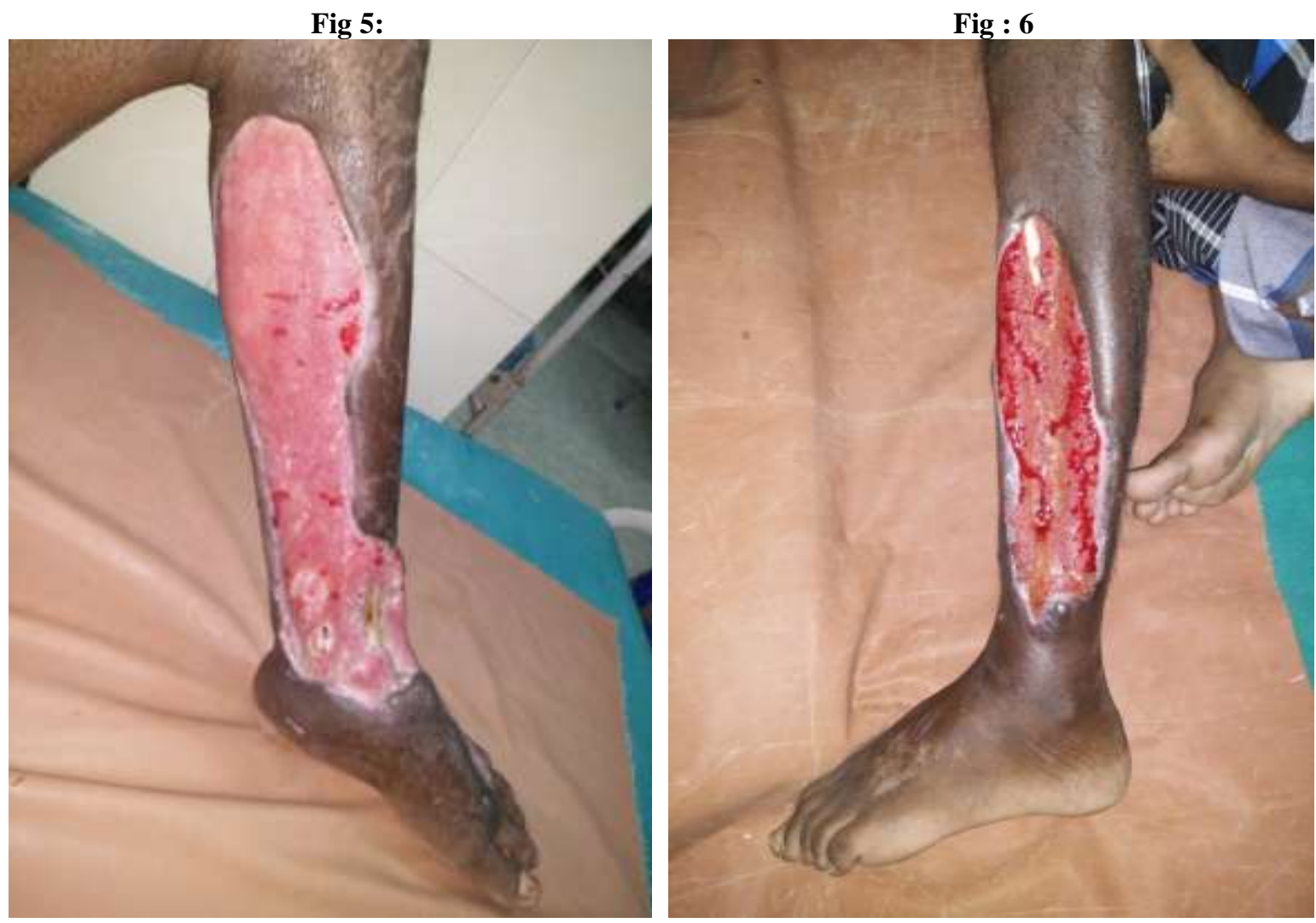

\section{Reference}

[1]. A chance to cut is not always a chance to cure- fasciotomy in the treatment of rattlesnake envenomation: A retrospective poison center study. Toxicon. 2015; 101:23-6 (ISSN: 1879-3150) Darracq MA; Cantrell FL; Klauk B; Thornton SL.

[2]. Is there a role for fasciotomy in Crotalinaeenvenomations in North America? Clin Toxicol (Phila). 2011; 49(5):351-65. Cumpston KL. Division of Clinical Toxicology, Department of Emergency Medicine, Medical College of Virginia Campus, Virginia Commonwealth University, 600 E. Broad St., Richmond, VA, 23112, USA. kcumpston@mcvh-vcu.edu

[3]. Elevated compartment pressures from copperhead envenomation successfully treated with antivenin. J Emerg Med. 2014; 46(1):34-7 (ISSN: 0736-4679) Mazer-Amirshahi M; Boutsikaris A; Clancy C.

[4]. Wound infections secondary to snakebite. GargA1, Sujatha S, Garg J, Acharya NS, Chandra Parija S.

[5]. Snakebite in Korea: A Guideline to Primary Surgical Management. Jung Ho Rha1, Sung Min Kwon 1, Jin Rok Oh 1 , ByungKeun Han 2, Kang Hyung Lee 3, and Jae Hyun Kim 1 Departments of 1 Orthopedics, 2 Internal Medicine, and 3 Emergency Medicine, Yonsei University Wonju College of Medicine, Wonju, Korea.

[6]. Hyperbaric oxygen therapy improves outcome of snake envenomation: tertiary center experience PradeothKorambayilMukundan1 ,PrashanthVarkeyAmbookan 1, Ragu Angappan 2 Department of Plastic Surgery, Jubilee Institute for Surgery of Hand, Aesthetic and Microsurgery, Thrissur 680005, Kerala, India. Department of Plastic Surgery, Sushruta Cosmetic and Laser Surgery Centre, Coimbatore 641004, Tamil Nadu, India.

[7]. Guidelines for the management of snake-bites -David A Warrell-World Health Organization, Regional Office for South-East Asia. 